\title{
The Impact of Stochastic Attenuation on Photometric Redshift Estimates
}

\author{
Thorsten Tepper García ${ }^{1}$ and Uta Fritze-von Alvensleben ${ }^{2}$ \\ ${ }^{1}$ Institut für Astrophysik, Georg-August Universität, \\ Friedrich-Hund-Platz 1, 37077 Göttingen, Germany \\ email: tepper@astro.physik.uni-goettingen.de \\ ${ }^{2}$ Centre for Astrophysics Research, University of Hertfordshire, \\ College Lane, Hatfield AL10 9AB, UK \\ email: ufritze@star.herts.ac.uk
}

\begin{abstract}
We model the stochastic attenuation by $\mathrm{HI}$ absorbers in the intergalactic medium (IGM), such as Ly $\alpha$ Forest clouds, and absorbers associated with galaxies, such as Lyman Limit systems (LLS) and Damped Lyman Alpha absorbers (DLAs), and compute an ensemble of $4 \cdot 10^{3}$ attenuated Spectral Energy Distributions (SEDs) in the Johnson system for the spectrum of a galaxy with a constant star formation rate (CSFR). Using these, we asses the impact of the stochastic attenuation on the estimates of photometric redshifts for this type of galaxy by comparison with model SEDs that include only a mean attenuation.
\end{abstract}

Keywords. galaxies: intergalactic medium, galaxies: high-redshift, galaxies: photometry

\section{Motivation}

The stochastic distribution of the (high column density) HI absorbers causes a significant scatter in the broadband colors of galaxies, especially at redshifts close to the drop-out redshift in the corresponding passband where the photoelectric absorption is more severe, and this is expected to drastically affect the estimates of photometric redshifts.

\section{Approach}

We model the attenuation due to Hi along a random line of sight (LOS) using differential distribution functions constrained from observations (Kim et al. 1997) in a Monte Carlo fashion (Bershady et al. 1999) as described in Tepper García \& Fritze-v.A. (in prep.). We generate an ensemble of $4 \cdot 10^{3}$ lines of sight out to a given redshift $z_{\text {em }}$, each of them containing a random absorber population. For each LOS we calculate an absorption mask, i.e. we compute the photoelectric and Lyman-Series line absorption (as yet just for the first five Lyman transitions) caused by each absorber for a flat input spectrum, modeling line profiles as in Tepper García (2006). We compute model galaxy spectra corresponding to a CSFR for redshifts in the range $0.0<z_{e m}<4.5$ using the Evolutionary Synthesis code GALEV (Bicker et al. 2004). For a given redshift, we multiply each of the $4 \cdot 10^{3}$ masks with our input spectrum and thus obtain an ensemble of an equal number of attenuated spectra. For each of these we compute a SED in the Johnson system. Using AnalySED (Anders et al. 2006) and a set of template SEDs that include only the mean attenuation (Madau 1995) for every redshift, we determine to which extent the redshifts of our simulated spectral energy distributions are recovered. 


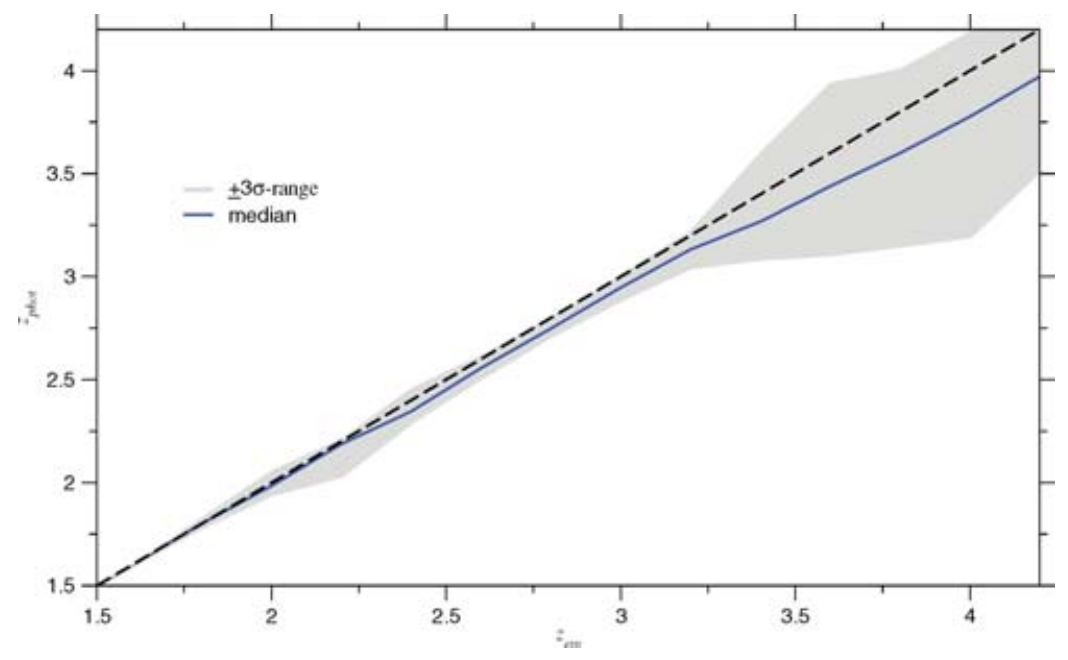

Figure 1. Estimate of the photometric redshift $z_{\text {phot }}$ for an Sd-type spectrum with stochastic attenuation, using as input the same spectrum with mean attenuation. The solid line shows the median, and the shaded area gives the $3 \sigma$-range around the mean for the entire ensemble of 4000 SEDs.

\section{Results \& Conclusions}

As can be seen in figure 1 , we find that, using the mean attenuation only, the median photometric redshifts are severe underestimated by typically $\Delta z_{p h o t}=0.3$, especially in the range $z>3.2$. In addition, we observe a substantial scatter of $\Delta z_{\text {phot }} \approx \pm 0.5$.

We predict that any method for determining photometric redshifts that only takes into account the mean attenuation at high redshifts should show a bias such as the one seen in figure 3. We hence emphasise the need for an accurate modeling of the attenuation including stochastic effects, in order to correctly interpret in combination with evolutionary synthesis models such as GALEV, the observations of high-redshift galaxies in deep multi-band imaging surveys.

\section{Acknowledgements}

TTG gratefully acknowledges support from the Georg-August-Universität, from CONACYT and the Astronomische Gesellschaft.

\section{References}

Anders, P., Bissantz, N., Fritze-v. Alvensleben, U., \& de Grijs, R. 2006, MNRAS, 347, 196

Bershady, M.A., Charlton, J.C., \& Geoffroy, J.M. 1999, ApJ, 518, 103

Bicker, J., Fritze-v.A., U., \& Fricke, K.J. 2004, A\&A, 413, 37

Kim, T.-S., Hu, E.M., Cowie, L.L., \& Songaila, A. 1997, AJ, 114, 1

Madau, P. 1995, ApJ, 441, 18

Tepper García, T. 2006, MNRAS, 369, 2025 